\title{
Cortical reactive balance responses to unexpected slippages while walking: a pilot study
}

\author{
Giovanni Mezzina, Federica Aprigliano, Silvestro Micera, Senior Member IEEE, Vito Monaco, and \\ Daniela De Venuto
}

\begin{abstract}
Understanding how the human brain cortex behaves when the dynamical balance is unexpectedly challenged can be useful to enable fall prevention strategies during daily activities. In this respect, we designed and tested a novel methodological approach to early detect modifications of the scalp-level signals when steady walking is perturbed.

Four young adults were asked to manage unexpected bilateral slippages while steadily walking at their self-selected speed. Lower limb kinematics, electromyographic (EMG) and electroencephalographic (EEG; 13 channels from motor and sensorymotor cortex areas) signals were synchronously recorded.

EMG signals from Vastus Medialis (both sides) were used to trigger the analysis of the EEG before and after the perturbation onset. Cortical activity was then assessed and compared pre vs. post perturbation. Specifically, for each gait cycle, the rate of variation of the EEG power spectrum density, named $m$, was used to describe the cortical responsiveness in five bands of interests: $\vartheta(4-7 \mathrm{~Hz}), \alpha(8-12 \mathrm{~Hz}), \beta$ I, $\beta$ II, $\beta$ III rhythms (13-15, 15-20, 18-28 Hz).

Results revealed a sharp increment of $m$ early after the onset of the perturbation (perturbed step) compared to steady locomotion, for all rhythms. This cortical behavior disappeared during the recovery step.

This study promisingly supports the evidence that the proposed approach can distinguish between steady walking and early reactive balance recovery, paving the way for the EEG-based monitoring of the fall risk during daily activities.
\end{abstract}

\section{INTRODUCTION}

Loss of balance, subsequent fall events and fall-related consequences show worrying numbers for the public health systems [1]: 1/3 (28-35\%) of the people aged $65+$ years falls at least once per year; the number of falls further increase up

This work was supported by the EU Commission through the $\mathrm{H} 2020$ project CYBERLEGs Plus Plus (The CYBERnetic LowEr-Limb CoGnitive Orthoprosthesis Plus Plus, Grant Agreement no. 731931), the Italian National Institute for Insurance against accidents at work (INAIL) within the MOTU project (PPR-AI 1-2), and the project AMICO (Assistenza Medicale In COntextual awareness, AMICO_Project_ARS01_00900).

D. De Venuto and G. Mezzina are with Politecnico di Bari, Dept. of Electrical and Information Engineering, Via E. Orabona 4, 70125, Bari, Italy. D. De Venuto is also with National Institute of Nuclear Physics (INFN).

F. Aprigliano, S. Micera, V. Monaco, are with The BioRobotics Institute, Scuola Superiore Sant'Anna, Viale Rinaldo Piaggio 34, 56025 Pontedera, Pisa, Italy. S. Micera is also with the Bertarelli Foundation Chair in Translational Neuro Engineering, Center for Neuroprosthetics and Institute of Bioengineering, School of Engineering, EPFL, Lausanne, Switzerland. V. Monaco is also with Fondazione Don Carlo Gnocchi, Milan, Italy. Corresponding author: G. Mezzina; phone: +39 0805963 562; email: giovanni.mezzina@poliba.it. to 5-7 in adults $70+$ years old. One over ten of those experiences serious injuries, such as hip fractures, subdural hematomas, head injuries etc. [2]. In addition, the subsequent "fear of fall" leads people to adopt a more sedentary lifestyle thus further increasing their risk of fall and reducing their overall quality of life [3].

To understand the physiological reasons underlying falling, some authors [4-8] investigated the cortical dynamics while subjects experienced perturbations, even unexpected, which could critically affect their orthostatic quite stance (e.g., stepping or foot-in-place from an orthostatic posture) [4]. They observed sharp changes in the cortical activity just after the perturbation (80-171 ms after stimulus) $[5,6]$. This behavior is firstly characterized by an increase of energy of the power spectral density (PSD) in the range $4-13 \mathrm{~Hz}(\vartheta$ and $\alpha$ waves) which is typically related to cognitive and sensorimotor functions (e.g., fall perception phase) [4]. In addition, it also shows an increase of energy of the PSD in the range $13-40 \mathrm{~Hz}$ ( $\beta$ I, II, III bands) mostly concerning motor functions [7-9]. Noticeably, after the recovery step, these cortical activity modifications are suppressed [4-9].

Other authors examined the cortical dynamics during steady walking and observed a significant causal unidirectional drive from the contralateral motor cortex to muscles of the swinging leg [10]. It is worth noting that the proactive modulation of cortical activity observed during walking is likely similar to that described in studies dealing with perturbed orthostatic posture [4, 10], though the responsiveness in the latter case (i.e., perturbation of orthostatic posture) is stronger than that observed in former one (i.e., steady walking).

Based on these evidences, we investigated changes in the cortical involvement when subjects were actively managing unexpected slippages delivered during steady walking. More in details, this work aims at developing, testing, and validating a strategy for the cortical dynamics information extraction in order to disentangle features related to reactive balance recovery from those concerning steady walking. Results confirm that the proposed approach allows for an early detection of the reactive behaviors, thus paving the way for the EEG-based monitoring of the fall risk during daily activities.

\section{MAteRIAL AND METHODS}

\section{A. Participants}

Four healthy young subjects ( 3 males, 1 female, $26.3 \pm 2.4$ years, $64.5 \pm 9.8 \mathrm{~kg}, 1.71 \pm 0.06 \mathrm{~m}$ ) were enrolled for this study. Before starting the experimental sessions, all participants signed an informed consent. Research procedures 


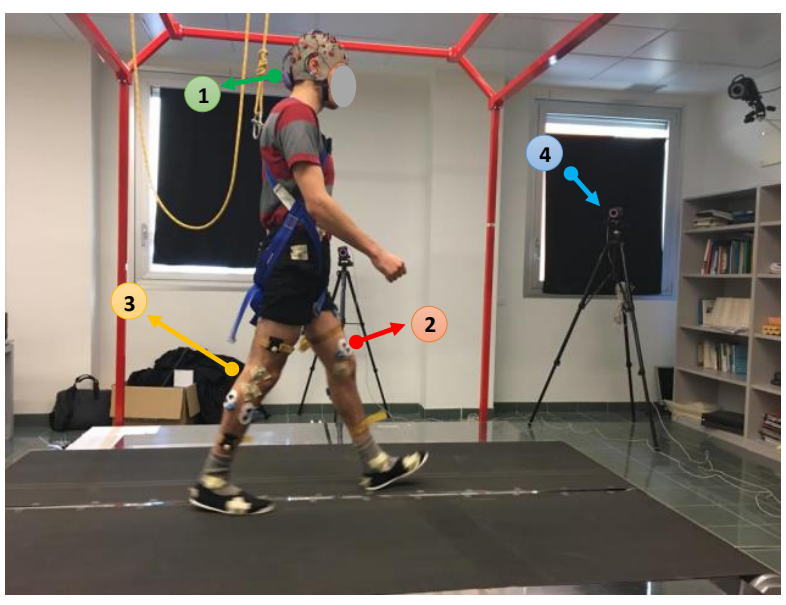

Figure 1. Experimental setup. The SENLY platform and a subject performing the test. On the figure, labels refer to: the wireless EEG headset (green - \#1), the wireless surface EMG electrodes (red - \#2), the set of markers (yellow- \#3) and the motion analysis system camera (blue \#4).

were in accordance with the Declaration of Helsinki and was approved by the Local Ethical Committee.

\section{B. Experimental Set-up}

Participants were asked to manage unexpected slippages while walking at their self-selected speed on a custom mechatronic platform named SENLY (Fig.1; [11]). They were secured by a safety harness attached to an overhead track. Volunteers donned a 32-channel wireless EEG headset (g.Nautilus Research by g.Tec), 10 wireless EMG surface electrodes (Cometa Wave Plus by Cometa Systems) and a set of 23 reflective markers for the 3D kinematics reconstruction, as shown in Fig. 1.

SENLY is a mechatronic platform designed to destabilize the balance control during different motor tasks [11-13]. It consists of a split-belts treadmill which belts can be independently moved in the horizontal plane, both longitudinally and transversally. It is also equipped with force sensors to identify the phases of the gait cycle during walking [13].

Thirteen EEG sites were monitored: F3, Fz, F4, C3, Cz, C4, Cp5, Cp1 Cp2, Cp6, P3, Pz, P4, according to the international 10-20 system [14]. The $\mathrm{O} 2$ electrode was used for noise suppression, $\mathrm{AFz}$ as ground and the A2 (right earlobe) as the reference electrode. Before each trial, an impedance check of all electrodes was carried out in order to ensure a value lower than $40 \mathrm{k} \Omega$. The EEG data were sampled at $500 \mathrm{~Hz}$ with 24-bit resolution [14].

Ten surface EMG channels were monitored from following bilateral muscle groups: Anterior Tibialis, Lateral Gastrocnemius, Vastus Medialis, Rectus Femoris, and Biceps Femoris. The EMG signals were recorded with a sample rate of $2048 \mathrm{~Hz}$ and down sampled to $500 \mathrm{~Hz}$ (16-bit resolution) to match the EEG signal sampling frequency [15]. For this study, only EMG signals related to the Vastus Medialis were retained.

Both EEG and EMG were transmitted via Bluetooth Low Energy protocol (BLE) to a dedicated gateway and collected by a Simulink model for further offline analysis [16].

To validate the temporal coherence of the EEG/EMG signals with the subjects' reactive response, the $3 \mathrm{D}$ trajectory of the markers located on suitable body landmarks of the lower limbs was recorded by an 8-camera-based system (Vicon Motion Analysis System - Oxford, U.K.) with a sample rate of $100 \mathrm{~Hz}$. Specifically, spherical markers $(\mathrm{d}=14 \mathrm{~mm})$ were mounted bilaterally on anterior superior iliac spines, sacrum, prominence of the greater trochanteres external surface, lateral and medial epicondyle of the femurs, heads of fibula, lateral and medial malleolus, calcaneus, first and fifth metatarsal heads. Additional markers were rigidly placed on wands over the midfemurs and midshaft of the tibia.

During the experimental sessions, kinematic records, electrophysiological signals and onset of the perturbation were synchronized on the same time line.

\section{Experimental protocol}

All involved subjects performed about $5 \mathrm{~min}$ of free walking in order to both find their preferred speed and allow them to acclimate. After that, participants underwent a series of 10 consecutive trials where their steady walking was unexpectedly perturbed by a slipping-like perturbation. Specifically, perturbations were delivered in a pseudorandomized fashion (i.e., five on the right foot and five on the left foot). Slipping-like perturbations consisted of a sudden and unexpected movement of one belt toward the anteroposterior (AP) direction starting when the heel strike of the foot being perturbed was detected. The perturbing belt was accelerated and decelerated to $0 \mathrm{~m} / \mathrm{s}$ by means of a triangular speed profile (slope $8 \mathrm{~m} / \mathrm{s}^{2}$ ) in order to cover a total displacement equal to $0.15 \mathrm{~m}$. The speed on the contralateral belt was maintained at the walking speed for the entire duration of the perturbation and then brought to zero.

\section{Data Pre-Processing}

EEG and EMG signals were collected from the same BLEinterfaced gateway and stored in a MATLAB workspace for further analysis. Recorded signals were initially filtered as follows:

- EEG. The EEGs were progressively band-filtered between $1 \mathrm{~Hz}$ and $40 \mathrm{~Hz}$ by using an $8^{\text {th }}$ order Butterworth filter before the transmission.

- EMG. Surface EMGs were on-line high-pass filtered with an $8^{\text {th }}$ order Butterworth filter with cut-off frequency at 10 $\mathrm{Hz}$ to reject movement artifacts.

- A numeric notch filters $48-52 \mathrm{~Hz}$ was implemented for both EEG and EMG signals.

- Kinematics. Each marker trajectory was low pass filtered (zero-lag, $4^{\text {th }}$ order Butterworth filter with cut-off frequency at $10 \mathrm{~Hz}$ ) and missing data were estimated by using cubic spline interpolation.

\section{E. Data Processing}

The data processing consisted of 2 main stages. Firstly, EMG signals were processed in order to identify the onset of each muscle contraction. The onset of the Vastus Medialis, namely muscle trigger (MT), was then used to enable the analysis of EEG signals for each gait cycle (both right and left sides). This latter step allowed us to investigate the cortical activity related to both the unperturbed gait cycles (i.e., strides before the onset of the perturbation) and the compensatory ones (i.e., strides after the perturbation onset). Fig. 2 summarizes the adopted algorithm. 

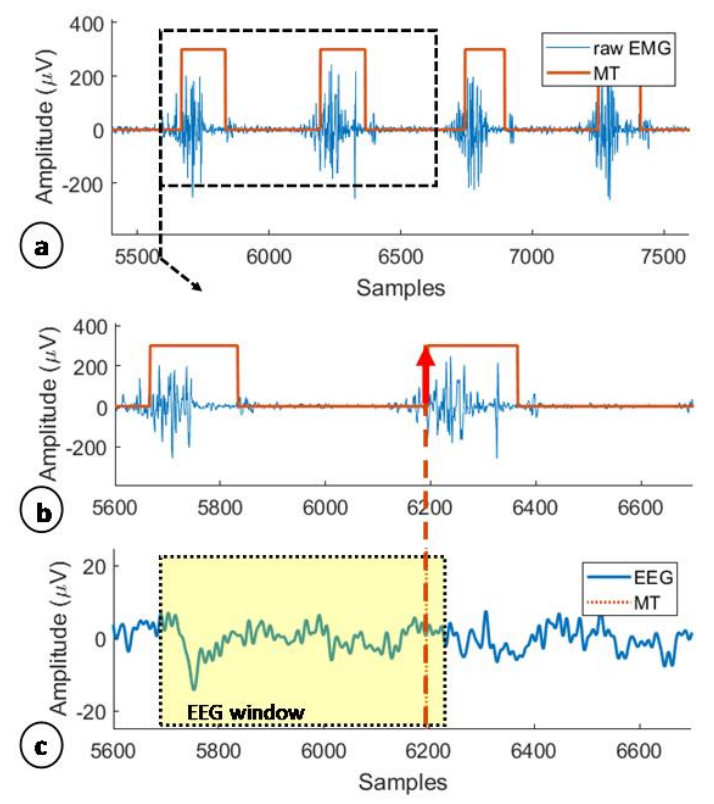

Figure 2. Overview of the technique timing and process: (a) the muscle trigger (MT) is extracted from the raw EMG; (b) detail of a specific MT rising edge (red arrow); (c) the MT rising edge enables an EEG window extraction (semi-transparent yellow area).

\section{1) EMG Muscle Trigger Generation}

Each EMG collected signal was rectified, squared, and stored in two shift registers. The first register stored 250 samples (i.e., $500 \mathrm{~ms}$ of acquisition) which were used to compute the average signal power, namely global average (GA). The second register stored the last 125 samples previously collected (i.e., $250 \mathrm{~ms}$ of acquisition) and were used to compute again the average signal power, namely local average (LA). This process was updated sample-bysample. For the $\mathrm{i}^{\text {th }}$ sample, the muscle was considered contracted if $\mathrm{LA}>\mathrm{GA}$. By using this approach, we converted an analog EMG signal to a binary one (Fig. 2, panels a and b).

\section{2) EEG Processing}

\section{a) Artifacts Mitigation Approach}

Once the rising edge of the Vastus Medialis (both sides) MT was detected, it enabled the EEG post-processing (Fig. 2). In particular, a $1.04 \mathrm{~s}$ (520 samples) long EEG time window was extracted across the MT as $1 \mathrm{~s}$ (500 samples) before and $0.04 \mathrm{~s}$ (20 samples) after the trigger. These values have been considered as optimal after a trial-and-error approach.

This subset of EEG data underwent an on-line Riemannian Artifact Subspace Reconstruction (rASR) [17]. The rASR is an online/offline artifacts attenuation method for mobile EEG data based on an ASR with Riemannian geometry. It was chosen because it can detect typical EEG artifacts while preserving the signal of interest, and it reduces the computational effort to one third if compared to the original ASR [17].

\section{b) EEG Responsiveness Estimation}

The rASR outcome was then split in 10 overlapped (2 samples) 500-samples long time windows, running along the whole chunk. For each of them, we computed the FFT and estimated the energy of the PSD in 5 bands of interests (BoI): $\vartheta(4-7 \mathrm{~Hz}), \alpha(8-12 \mathrm{~Hz}), \beta$ I, $\beta$ II, $\beta$ III rhythms $(13-15,15-$ $20,18-28 \mathrm{~Hz}$ ). Overall, a 10x5 matrix was extracted for each of the thirteen channel, where 10 and 5 refer to the number of time windows and BoI, respectively.

For each BoI, a linear model (ordinary least squares (OLS) method) fitting data related to the 10 time windows was computed. The slope of this model, namely $m$, was used to describe the cortical responsiveness. Specifically, according to the literature [4-9], a sharp increment of $m$ documents an increase of the PSD energy content, thus reflecting a greater involvement of the cortical area.

The $m$ trend across the onset of the perturbation was assessed in three different periods: steady walking, early lack of balance (eLoB) and recovery step. Data related to steady walking refer to a set of 5 unperturbed steps (i.e., before the onset of the perturbation). The eLoB represents the compensatory step (from the onset of the perturbation to the first heel strike of the unperturbed foot). The recovery step starts with the first heel strike of the unperturbed foot and ends with the heel strike of the contralateral foot (i.e., perturbed limb).

\section{F. Statistical Analysis}

For each experimental trial, the outcome variable (i.e., $m$ ) was used as dependent measure.

A 3-way repeated measures ANOVA was performed to determine the main effect of the EEG channels (13 levels: F3, Fz, F4, C3, Cz, C4, Cp5, Cp1 Cp2, Cp6, P3, Pz, P4), BoI (5 levels: $\vartheta, \alpha, \beta$ I, II, III bands) and observed phases (3 levels: walking, eLoB and recovery step).

\section{EXPERIMENTAL RESULTS}

Subjects walked at an average speed of $1.07 \pm 0.07 \mathrm{~m} / \mathrm{s}$ (range: $1-1.15 \mathrm{~m} / \mathrm{s}$ ). No falls were reported during the trials, since all participants were always able to recover their balance.

Fig. 3 shows a representative dataset concerning the estimated $m$ for each BoI and channel (vertical axis) along all Vastus Medialis MTs.

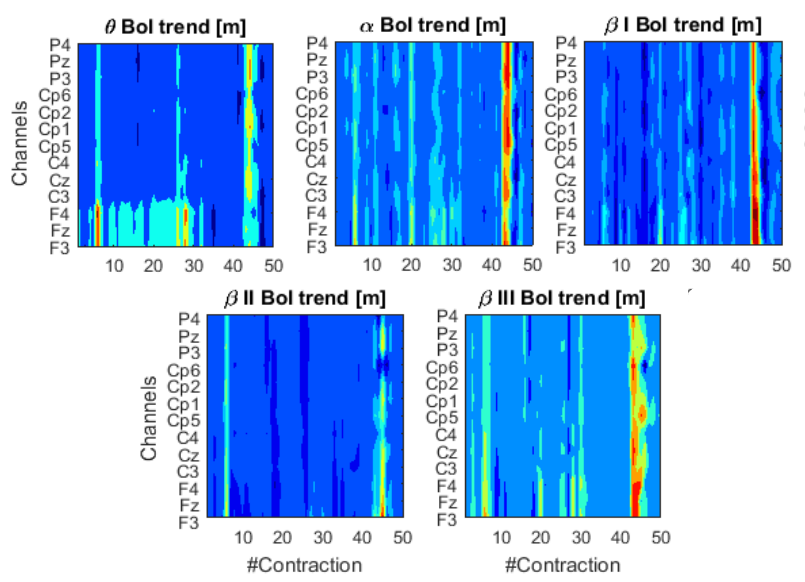

Figure 3. OLS-based estimation of the $m$ values for all the analyzed BoI. The showed data are extracted from the dataset: Sub. 4 - Trial 2. The colorbar of each subplot is normalized to local $m$ minimum (blue) and maximum (red). All panels show that $m$ contextually increases just after the 41 st muscle contraction, when the slippage was delivered. 


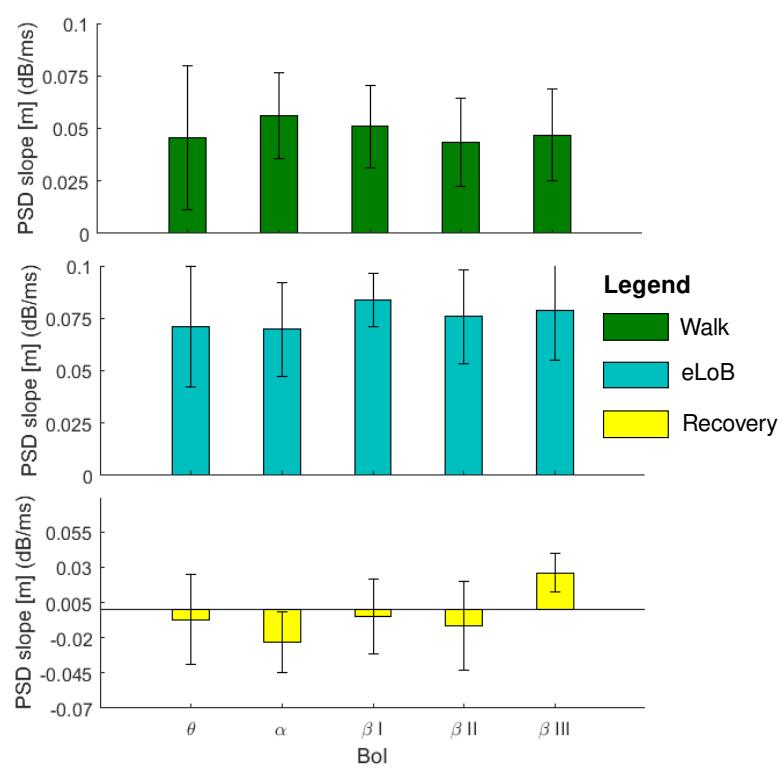

Figure 4. $m$ values (mean \pm std across subjects) versus the BoI. Subplots show the $m$ parameter in three monitored phases.

Noticeably, all panels related to each BoI show that $m$ sharply increases just after the $41^{\text {st }}$ muscle contraction, when the perturbation was delivered.

Statistical analysis revealed that $m$ was mainly influenced by the BoI $(\mathrm{p}=0.004)$ and the observed phases $(\mathrm{p}<0.0001)$.

In particular, $m$ resulted significantly lower during the recovery step than during the walking and $\mathrm{eLoB}$ phases, as reported in Fig. 4. No main effect was observed across EEG channels $(\mathrm{p}=0.502)$.

Fig. 4 shows that the $\beta$ rhythms experienced the highest relative difference $\Delta m$ passing from the walking phase to the one immediately following (i.e., walking $\rightarrow \mathrm{eLoB}$ ).

Considering all the $\beta$ rhythms the $\Delta m_{\text {walk-eLoB }}=+0.0325 \pm$ $0.005 \mathrm{~dB} / \mathrm{ms}$. Fig. 4 also shows that the $\alpha$ rhythm reflected the highest $\Delta m$ passing from the eLoB to the recovery phase $\Delta m_{e L o B-r e c}=-0.0928 \mathrm{~dB} / \mathrm{ms}$.

\section{DISCUSSION AND CONCLUSION}

The aim of this study has been the investigation of the cortico-muscular dynamics in a high postural demand situation, in which participants underwent sudden and unexpected slippages delivered during steady walking. We introduced a new inspection methodology based on the linear OLS estimation for the EEG responsiveness assessment, which allowed us to track PSD energy trend immediately after a specific muscle activation.

The results confirmed the expected trend $[4,10]$, showing a lower $m$ during steady walking if compared with that's estimated during the eLoB. These differences were observed for all BoIs, with a strong incidence on frequencies higher than $13 \mathrm{~Hz}$, which concern to motor functions [7-9].

Overall, despite the small number of subjects, these results promisingly support the evidence that the proposed approach can distinguish between steady walking and early reactive balance recovery. In this respect, to the best of our knowledge, this is the first study in which the effects of slipping-like perturbations during walking, in terms of scalplevel cortical dynamics, are investigated.

The proposed method was actually designed to be computationally efficient. It is hence expected to be suitable for real time applications involving detection of lack of balance and enabling of mitigation strategies mediated by wearable robotic platforms. However, this hypothesis deserves further enquiries, which are - at the moment - under investigation.

\section{REFERENCES}

[1] World Health Organization. "WHO global report on falls prevention in older age.", 2008. Online updated: Jan. 16, 2018

[2] Bartl, R., and Bartl, C. "Falls and Fall Prevention" Osteoporosis Manual. Springer, Cham, pp. 263-263, 2019.

[3] Blain, H. et al. "Effectiveness of a programme delivered in a falls clinic in preventing serious injuries in high-risk older adults: A preand post-intervention study". Maturitas, 122, pp. 80-86, 2019

[4] Solis-Escalante, T. et al. "Cortical dynamics during preparation and execution of reactive balance responses with distinct postural demands." NeuroImage, 188, pp. 557-571, 2019.

[5] Wittenberg E. et al. "Neuroimaging of Human Balance Control: A Systematic Review". Front. Hum. Neurosci. 11:170, 2017. doi: 10.3389/fnhum.2017.00170"

[6] Varghese, J. P., et al. "Standing still: is there a role for the cortex?". Neuroscience letters, 590, pp. 18-23, 2015

[7] Cavanagh, J.F., Frank, M.J. "Frontal theta as a mechanism for cognitive control." Trends Cognit. Sci. 18, pp. 414-421, 2014.

[8] Freyler K., et al. "Reactive Balance Control in Response to Perturbation in Unilateral Stance: Interaction Effects of Direction, Displacement and Velocity on Compensatory Neuromuscular and Kinematic Responses". PLOS ONE 10(12): e0144529, 2015.

[9] Mierau, A., et al. "Cortical correlates of human balance control" Brain topography, 30(4), pp. 434-446, 2017.

[10] Artoni, F., et al. "Unidirectional brain to muscle connectivity reveals motor cortex control of leg muscles during stereotyped walking." Neuroimage, 159, pp. 403-416, 2017.

[11] Bassi Luciani L., Genovese V., Monaco V., Odetti L., Cattin E., and Micera S., "Design and evaluation of a new mechatronic platform for assessment and prevention of fall risks," Journal of Neuroengineering and Rehabilitation, 9.1, pp. 51, 2012.

[12] Aprigliano F., Martelli D., Tropea P., Pasquini G., Micera S., and Monaco V. "Aging does not affect the intralimb coordination elicited by slip-like perturbation of different intensities". Journal of neurophysiology, 118(3), pp. 1739-1748, 2017.

[13] Martelli D., Aprigliano F., Tropea P., Pasquini G., Micera S., and Monaco V. "Stability against backward balance loss: Age-related modifications following slip-like perturbations of multiple amplitudes". Gait \& Posture, 53, pp. 207-214, 2017.

[14] De Venuto D., Annese V. F., Ruta M., Di Sciascio E. and Sangiovanni Vincentelli A. L. "Designing a Cyber-Physical System for Fall Prevention by Cortico-Muscular Coupling Detection,". IEEE Design \& Test, 33(3), pp. 66-76, June 2016. doi: 10.1109/MDAT.2015.2480707

[15] Annese V. F., Crepaldi M., Demarchi D. and De Venuto D. "A digital processor architecture for combined EEG/EMG falling risk prediction," Design, Automation \& Test in Europe Conference \& Exhibition (DATE), Dresden, 2016, pp. 714-719, 2016.

[16] de Tommaso M., Vecchio E., Ricci K., Montemurno A., De Venuto D. and. Annese V. F. "Combined EEG/EMG evaluation during a novel dual task paradigm for gait analysis," 6th International Workshop on Advances in Sensors and Interfaces (IWASI), Gallipoli, pp. 181-186, 2015. doi: 10.1109/IWASI.2015.7184949

[17] Blum S., Bleichner M. G., Debener S. "Evaluation of Riemannian Artifact Subspace Reconstruction for the correction of EEG artifacts". Frontiers in Human Neuroscience. N. 00134, 2019. DOI $=10.3389 /$ conf.fnhum.2018.227.00134 